\title{
Preventing venous thromboembolism at a district hospital: a quality improvement study
}

\section{Bernhard G Beutel \& Louis S Jenkins}

To cite this article: Bernhard G Beutel \& Louis S Jenkins (2015) Preventing venous thromboembolism at a district hospital: a quality improvement study, South African Family Practice, 57:2, 64-68, DOI: 10.1080/20786190.2014.977033

To link to this article: http://dx.doi.org/10.1080/20786190.2014.977033
(c) 2015 The Author(s). Open Access article distributed under the terms of the Creative Commons License [CC BY-NC-ND 4.0]
曲 Published online: 18 Feb 2015.

Submit your article to this journal $\pi$

Џll Article views: 235

Q View related articles $\sqsubset$

View Crossmark data \lceil 


\title{
Preventing venous thromboembolism at a district hospital: a quality improvement study
}

\author{
Bernhard G Beutel ${ }^{a, b}$ and Louis S Jenkins ${ }^{c *}$ \\ a Postnet Suite 13, Private Bag X680, Oudtshoorn, 6620, South Africa \\ ${ }^{b}$ George Provincial Hospital, George, Eden District, Western Cape, South Africa \\ 'Division of Family Medicine and Primary Health Care, Faculty of Health Sciences, Stellenbosch University, Stellenbosch, South Africa \\ *Corresponding author, email:Louis.Jenkins@westerncape.gov.za
}

Background: Pulmonary embolism (PE) is the most common preventable cause of hospital deaths, and almost all hospitalised patients have at least one risk factor for venous thromboembolism (VTE). Despite the availability of highly effective thromboprophylaxis in preventing VTE, numerous studies worldwide have demonstrated its under-utilisation. The aim of this study was to review and improve the utilisation of thromboprophylaxis in the prevention of VTE in hospitalised patients at Oudtshoorn district hospital, and to make recommendations to the Western Cape Department of Health on how to improve the quality of care for patients at risk for VTE at district hospitals.

Method: A quality improvement cycle (QIC). Retrospective analysis of files of adult patients admitted to the male and female wards at Oudtshoorn district hospital was performed prior to and after a five-month intervention phase. The target standards for the QIC were: (1) availability of a written hospital policy on VTE prevention; (2) every adult admission should have a formal VTE risk assessment documented; (3) every adult admission who is at risk for VTE should receive thromboprophylaxis.

Results: Some $38 \%$ of adult patients admitted to Oudtshoorn Hospital, excluding the maternity ward, were at risk of developing VTE. There was no written hospital policy on VTE prevention. This was developed and made available during the intervention. In the pre-intervention group there were no patients who had a documented VTE risk assessment. The post intervention group showed a considerable increase with $45.2 \%$ having had a completed VTE risk assessment on admission $(p<0.001)$. In the pre-intervention group only $4.6 \%$ of patients who were at risk of VTE received thromboprophylaxis. There was a statistically significant difference in the number of patients at risk who received thromboprophylaxis in the post-intervention group where $36 \%$ of these patients received thromboprophylaxis $(p<0.001)$.

Conclusions: The study identified a major shortcoming in the prevention of VTE in those patients at risk who were admitted to Oudtshoorn district hospital. An intervention as part of a quality improvement cycle has been able to demonstrate a significant improvement in the detection of patients who are at risk of VTE and a subsequent improvement in appropriate thromboprophylaxis. A number of barriers to their implementation have been identified and need to be addressed. This QIC may in time be of value to assist other district hospitals in addressing the issue of VTE prevention.

Keywords: district hospital, quality improvement, venous thromboembolism prevention

\begin{abstract}
Background
Pulmonary embolism (PE) is the most common preventable cause of hospital deaths and is estimated to be the cause of approximately $10 \%$ of all deaths in hospitalised patients. ${ }^{1,2}$ In the United Kingdom a recent parliamentary report noted up to 25000 deaths per year from venous thromboembolism (VTE) associated with hospitalisation. ${ }^{3}$
\end{abstract}

Despite these alarming statistics, various international studies have demonstrated the under-utilisation of thromboprophylaxis for those patients who are at risk of developing VTE. ${ }^{4}$ This is not limited to the developing world as studies in Canada found that less than $25 \%$ of at-risk hospitalised patients received thromboprohylaxis. ${ }^{4} \mathrm{~A}$ multitude of randomised control trials over many years have demonstrated that thromboprophylaxis is highly effective in preventing VTE and PE. ${ }^{1}$ Almost all hospitalised patients have at least one risk factor for VTE and about $40 \%$ have three or more risk factors. ${ }^{1}$ A multinational cross-sectional study of hospitalised patients showed that on average 52\% (range 35\% to $73 \%$ ) were at risk of VTE. ${ }^{5}$ Therefore, a significant proportion of in-patients are at high risk of VTE and are not being protected against it.

In the United States, hospital-related PE has been ranked as the single most important intervention to improve patient safety in hospitals. ${ }^{6}$ The morbidity and mortality of VTE is significant. A single deep vein thrombosis (DVT) is associated with a vastly increased risk of recurrent events and long-term complications, which include post-thrombotic syndrome and pulmonary hypertension. ${ }^{7}$ These patients require long-term anticoagulation with its associated risks and costs. The annual costs involved with a single VTE event in the US have been estimated to be between \$ 7594 and \$ 16644 . $^{7}$

A number of international studies have attempted to bridge the gap between the established effectiveness of thromboprophylaxis and its clinical implementation. These studies have revolved around two main concepts. The first concept focused on which risk assessment models were accurate and user-friendly. These various models attempt to classify the degree of risk of VTE and are usually divided into medical and surgical models. The Southern African Society of Thrombosis and Haemostasis published the "Venous thromboembolism: Prophylactic and therapeutic practice guideline" in 2009, with a recent update in April 2013., It contains a user-friendly table of VTE risk subcategories, which is combined for surgical and non-surgical patients. ${ }^{2,8}$ This guideline provides a practical tool for risk assessment as well as recommendations on the means of thromboprophylaxis, but not measures to improve the rate of VTE prevention. 
Second, studies have looked to identify strategies to increase the use of thromboprophylaxis in at-risk patients. They all support active initiatives as opposed to passive (e.g. distribution of guidelines alone) with the most successful strategies being multifaceted and multidisciplinary. The interventions include a formal hospital policy with organisational change, various doctor reminders and decision support systems, staff education programmes, and regular audit processes.

There are currently no data on the incidence of VTE in South Africa. The aim of this study was to review and improve the utilisation of thromboprophylaxis in the prevention of VTE in hospitalised patients at Oudtshoorn district hospital, where this problem has been noted. The objectives were to assess the current quality of thromboprophylaxis; to plan and implement quality improvement measures; to determine if these were associated with a significant improvement in the quality of care; and to make recommendations to the Western Cape Department of Health on how to improve the quality of care for patients at risk for VTE at district hospitals.

\section{Methods}

\section{Study design}

The quality improvement cycle (QIC) involves a cycle consisting broadly of setting target standards; monitoring current performance and comparing it with the target; effecting change and assessing its effectiveness to improve the quality of care (see Figure 1).

\section{Setting}

Oudtshoorn provincial hospital is a district hospital with 123 beds in the Eden district of the Western Cape, South Africa, serving a population of approximately 60000 people. It has four wards (adult male, adult female, maternity, paediatric), an emergency centre, outpatient department and an anti-retroviral treatment clinic. The medical staff consists of three community service doctors and seven medical officers. There is an average of 50 admissions per week to the general adult wards.

In addition to general medical admissions, the hospital performs various elective level 1 surgical procedures, including tonsillectomies, circumcisions and caesarean sections. Emergency surgeries performed include laparotomies for ectopic pregnancies and appendicectomies. Visiting specialist disciplines include internal medicine, family medicine, obstetrics and gynaecology, surgery, orthopaedics, ENT and ophthalmology.

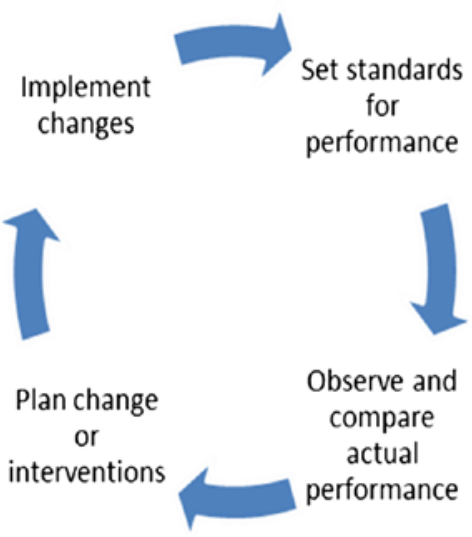

Figure 1: Steps of a quality improvement cycle

\section{Sampling and data collection}

Data were collected by the author at the beginning of 2012 from 250 patient files. The sample consisted of consecutive admissions to the adult wards split evenly between the male and female wards. This was done retrospectively by checking the admission entries in the ward admission registers and was intended to prevent bias, which might have occurred if the clinical team had been aware of an audit taking place. The sample size was determined with the help of the Department of Statistics at Stellenbosch University. Patient files were examined for the following variables:

(1) Was a formal risk assessment done during admission?

(2) What sub-category of VTE risk (low, moderate, high) was the patient?

(3) Was thromboprophylaxis provided if at risk?

Patients were assigned to sub-categories of VTE risk depending on a number of variables obtained from their files. These included age, indication for admission, comorbidities, current medications and duration of surgery, among others. These variables were used in conjunction with the "Venous thromboembolism: prophylactic and therapeutic practice guideline" of the Southern African Society of Thrombosis and Haemostasis to assess the risk category of the patient. ${ }^{2}$

From these data the actual performance of the hospital in relation to each of the pre-determined criteria was analysed, and captured in an Excel spreadsheet.

\section{Setting target standards}

Almost all research and guidelines on this topic originate from a developed world setting. The American College of Chest Physicians (ACCP) recommends that every hospital develop a written policy and an active strategy to prevent VTE.' In the United States the National Quality Forum and the Joint Commission both recommend that a formal risk assessment evaluation for VTE should be done on each patient on admission and regularly thereafter. Locally, the guidelines of the Southern African Society of Thrombosis and Haemostasis provide "clinical" standards focused on the desired outcomes of care, but not structural or process standards to improve the rate of appropriate thromboprophylaxis prescription. ${ }^{2}$ The target standards used for this QIC were as follows:

\section{Structural}

A written hospital policy on VTE preventions. This included the various strategies needed to provide quality thromboprophylaxis.

Process

Every adult admission should have had a formal VTE risk assessment documented.

\section{Outcome}

Every adult admitted who was at risk for VTE should have received appropriate thromboprophylaxis. This may have taken the form of pharmacological or non-pharmacological means.

\section{Comparing actual performance with target standards}

The analysed data were compared with the target standards set out above.

\section{Planning changes to improve quality}

The interventions included a formal hospital policy with organisational changes, various doctor reminders and decision support systems, staff education programmes, and regular audit processes. Various studies including a systematic review of 


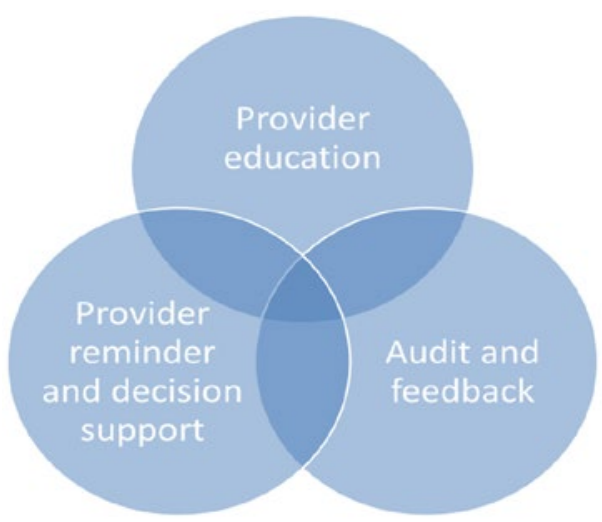

Figure 2: Intervention strategies used in the study

interventions to improve prophylaxis for VTE have found that a number of active strategies implemented together are most likely to be successful in achieving optimal outcomes. ${ }^{9}$

In a discussion that the author had with the hospital manager, medical manager, most of the hospital doctors, male and female ward nursing managers and senior pharmacist a number of interventions were agreed upon that would be implemented to improve the quality of thromboprophylaxis (see Figure 2).

\section{Implementing change}

This took place over a five-month period from July to November 2012.

\section{Provider education}

This included regular interaction with doctors and nursing staff on the importance of VTE prevention, the use of the risk assessment tool provided and the correct utilisation of thromboprophylaxis options.

An initial interactive teaching session was held, which provided the results of the current performance and compared it with the target standards. During this session the risk assessment tool was introduced and its use explained with the use of various patient scenarios. Follow-up sessions were held with the doctors and nursing staff. These were intended to address concerns and uncertainties as well as to reinforce the clinical importance of the intervention.

\section{Provider reminder and decision support}

A stamp was adapted from a VTE intervention used by the Salisbury NHS Foundation Trust in Salisbury, United Kingdom to include the risk subcategories of the Southern African Society of Thrombosis and Haemostasis VTE guidelines (see Figure 3). ${ }^{10}$ It was placed on the back of the prescription chart where doctors' orders were written and served to remind doctors to assess patients' risk on admission and to prescribe appropriate thromboprophylaxis if indicated. If heparin was contraindicated in patients at risk of VTE the reason needed to be specified on the stamp. The nursing staff were requested to check whether the stamp had been completed. If not, they were to notify the ward doctor.

The risk assessment tool of the Southern African Society of Thrombosis and Haemostasis was chosen for the intervention due to its ease of use and because it takes into account the local context with its high burden of human immunodeficiency virus (HIV) and tuberculosis (TB), which most international tools fail to include. The fact that HIV infection causes a hypercoaguable state is well documented, with the prevalence of VTE in the HIV-infected population estimated to be as high as $18 \% .^{11,12}$

The tool was made available in the form of posters, which were placed at each desk in the emergency centre and outpatients department as well as at the work-stations of the adult wards. They were placed in a manner so as to be easily visible during the admission process of patients. Laminated A4 cards were also placed at each of these sites and on the ward round trolleys of the male and female wards.

\section{Audit and feedback}

An audit was performed at five months post-intervention (see data collection post-intervention below). It is recommended that this is repeated six-monthly by the medical manager and/or family physician with feedback to the clinical staff.

\section{Hospital policy on VTE prevention}

Together with the hospital manager, a policy was drawn up entitled: Oudtshoorn Hospital Policy on Venous Thrombo-Embolism (VTE) Prevention. Its stated purpose was to establish an effective, sustainable approach to the prevention of VTE in adult patients admitted to Oudtshoorn Hospital.

\section{Data collection post-intervention}

A repeat retrospective analysis was conducted five months after the initiation of the intervention to determine whether the prevalence of correct thromboprophylaxis administration had

\begin{tabular}{|c|c|c|c|c|}
\hline \multicolumn{5}{|c|}{ VTE RISK ASSESSMENT } \\
\hline \multicolumn{2}{|c|}{ Circle Risk category } & Lov & Moderate & High \\
\hline \multirow{2}{*}{$\begin{array}{l}\text { Prophylaxis } \\
\text { type indicated } \\
\text { (Circle and } \\
\text { prescribe on } \\
\text { drug chart) }\end{array}$} & \multicolumn{2}{|c|}{ Heparin } & \multicolumn{2}{|c|}{ If heparin not indicated specify reason below } \\
\hline & \multicolumn{2}{|c|}{$\begin{array}{l}\text { Compressive } \\
\text { stockings }\end{array}$} & & \\
\hline \multicolumn{3}{|l|}{ Signature: } & Date: & \\
\hline
\end{tabular}


changed. An additional sample of 199 patient files was assessed retrospectively for the same variables, and included consecutive admissions to the male and female wards.

\section{Results}

The study participants consisted of 250 adults in the pre- and 199 adults in the post-intervention groups. There were equal numbers of male and female patients in the pre-intervention group. The post-intervention group showed a $59 \%$ male to $41 \%$ female split.

In the pre-intervention group there were no patients who had a documented VTE risk assessment. The post-intervention group showed a considerable increase with 90 patients (45.2\%) having had a completed VTE risk assessment stamp on the admission chart $(p<0.001)$ (see Figure 4).

Patients are deemed at risk of VTE if they fall into the moderate- or high-risk categories. There was no statistically significant difference in the number of patients at risk of VTE between the pre- and

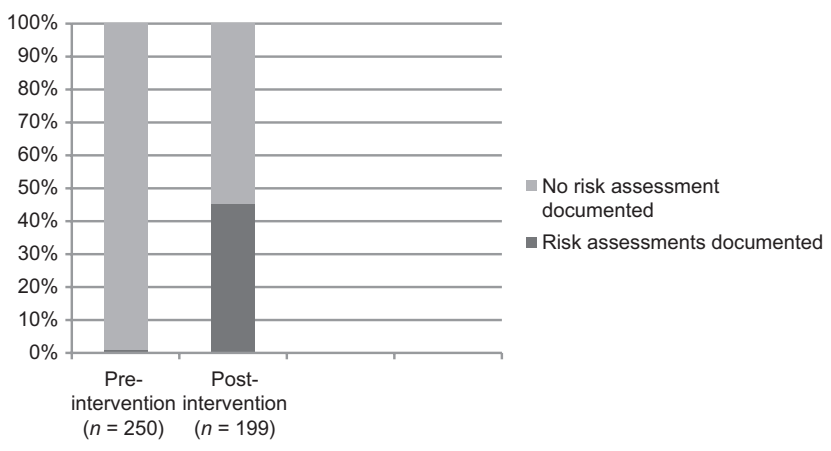

Figure 4:VTE risk assessments documented in patient notes on admission

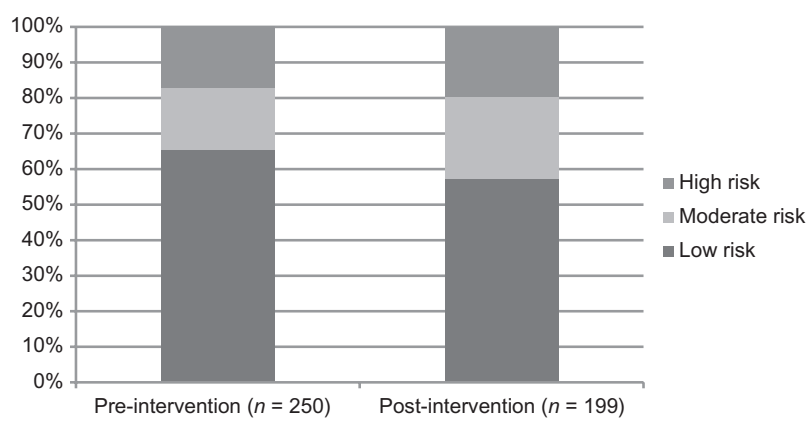

Figure 5: Risk categories of patients admitted

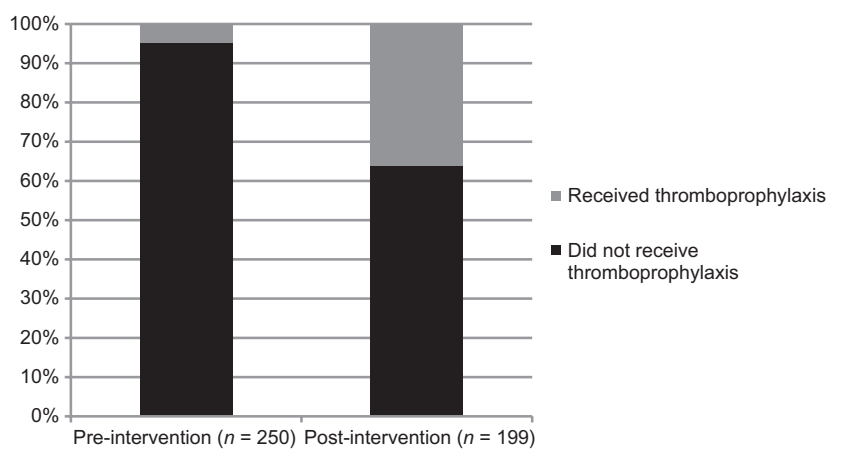

Figure 6: Percentage of patients at risk of VTE who received thromboprophylaxis post-intervention groups $(p=0.079)$. The pre-intervention group included 86 patients at risk (34.4\%) and the post-intervention group included 84 patients (42.7\%). The reasons for this small difference between the two groups were not clear from the data (see Figure 5).

In the pre-intervention group, 86 patients were at risk of VTE. Of these only four patients received thromboprophylaxis (4.6\%). There was a statistically significant difference in the number of patients at risk who received thromboprophylaxis in the post-intervention group where 31 of the 85 patients (36\%) at risk received thromboprophylaxis $(p<0.001)$ (see Figure 6 ).

\section{Discussion}

A large proportion of adult patients admitted to Oudtshoorn hospital were at significant risk of developing VTE. The average percentage in the pre- and post-intervention groups showed that $38.5 \%$ of all adult admissions were at moderate to high risk. This equates to slightly more than one in three admissions. It is not known how many of these patients actually developed either a DVT or PE. It is very likely that other district hospitals in the Western Cape which have comparable patient demographics and burden of disease would have similar percentages of patients at risk of VTE.

International data suggest a higher VTE risk than we found with this study. This may be due to an under-estimation of the actual risk, as the calculation of risk was done retrospectively by reviewing patient files. Various risk factors could not be easily elicited such as the body mass index (BMI) of the patient and a past history of DVT, which is not routinely asked and recorded in patient notes. We assessed risk only on the basis of available data in the patient file. It may also be due to the use of different risk assessment guidelines. The ENDORSE multinational cross-sectional study of 358 hospitals in 32 countries found that on average $52 \%$ of adult patients were at risk of VTE (range 35-73\%). This was based on the American College of Chest Physicians consensus guidelines. ${ }^{5}$ Utilising these same guidelines a cross-sectional survey of 12 hospitals in Senegal found that $57 \%$ of patients were at risk of VTE. Both of these studies included medical admissions above the age of 40 and surgical admissions above 18 years of age. ${ }^{13}$

Prior to this intervention there was no strategy to prevent VTE at Oudtshoorn Hospital. The use of thromboprophylaxis was haphazard and was often only the continuation of treatment in a patient referred back from specialist care at the local referral hospital. There were no structured means of identifying patients who were at risk. This is clear from the fact that no patients had a formal VTE risk assessment, and that only $4.6 \%$ of patients at risk of VTE received thromboprophylaxis.

The under-utilisation of thromboprophylaxis is a worldwide problem that is not limited to the developing world. Alarmingly, in the pre-intervention group of this study the rate of appropriate thromboprophylaxis use (4.6\% of patients at risk of VTE) was well below that cited internationally, where $58.5 \%$ of surgical and $39.5 \%$ of medical patients at risk received thromboprophylaxis. ${ }^{5}$ Data from another sub-Saharan country showed that $33.8 \%$ of medical and $37.5 \%$ of surgical patients at risk of VTE received thromboprophylaxis. ${ }^{13}$

The quality improvement intervention in our study led to a statistically significant improvement in the number of risk assessments done $(p<0.001)$ and the percentage of patients at risk who received thromboprophylaxis $(p<0.001)$. This should be seen in light of the very poor performance before the intervention. It is, however, an indication that improvement is 
possible, and this could form the basis of an ongoing quality improvement programme. The intervention was based on a number of strategies that have been employed in quality improvement programmes internationally. Active quality improvement strategies in various hospitals in the UK based on similar interventions showed improvements in the rates of appropriate thromboprophylaxis from $59 \%$ to $70 \%(p<0.05)$ and from $25 \%$ to $77 \%(p<0.05)$ respectively. ${ }^{14}$

This QIC has determined target standards that can be used for follow-up audits to monitor performance. This is important in maintaining a sustainable intervention that has been shown to be effective. There are a number of barriers that have been identified and which can be overcome.

\section{Limitations of this study}

For the majority of the study and for the full duration of the intervention the author was working at the local referral hospital. This made it difficult to provide a consistent drive to the intervention in terms of motivating and reminding the clinical team of the need to assess patients on admission for their VTE risk. The presence of such a person might have resulted in further improvements in outcomes. A dedicated person, most likely the medical manager or family physician, will be needed to carry this quality improvement programme forward to ensure future sustainable improvement.

There were a number of further logistical factors that limited the intervention. These included the presence of several locum doctors working at the hospital as well as visiting specialists. It was difficult for the author not being on site to involve these clinicians. As part of a continued intervention they need to be introduced to this aspect of clinical care at the hospital and familiarised with the local protocols. Unfortunately not all doctors who work at the hospital attended the educational meetings that formed part of the intervention.

As mentioned earlier, the retrospective analysis of patient files was a methodological weakness of this study. Prospective analysis, however, would have required patient consent with the potential for bias as patients being aware of the intervention would have placed pressure on clinicians to prescribe thromboprophylaxis, which may have skewed the results. ${ }^{15}$

\section{Conclusion}

In all, $38 \%$ of adult patients admitted to Oudtshoorn hospital (excluding the maternity ward) were at risk of developing VTE. This study identified a major shortcoming in the prevention of VTE in these patients. An intervention as part of a quality improvement cycle was able to demonstrate a significant improvement in the detection of patients who are at risk of VTE and a subsequent improvement in the administration of appropriate thromboprophylaxis. Much still needs to be done to bring the quality of VTE prevention to that of the study target standards. However, these targeted interventions have shown the potential to make a difference. A number of barriers to their implementation have been identified and will need to be addressed. This QIC may in time be of value in assisting other district hospitals to address the issue of VTE prevention.

\section{Ethical Approval}

Stellenbosch University Health Ethics Committee reference number N11/07/221. The study was conducted according to the principles of the Declaration of Helsinki (Seoul version, 2008).

Acknowledgements - Thanks are offered to the staff at Oudtshoorn Hospital for their valuable assistance in the planning and implementation of the intervention, in particular to the admission clerks for the retrieval of the files, to the author's supervisor, Dr Louis Jenkins for his guidance and encouragement, and to Mr Justin Harvey at the Centre for Statistical Consultations at Stellenbosch University for his assistance with the sample size calculations and statistical analysis of the study outcomes.

Conflict of interest - None.

\section{References}

1. The American College of Chest Physicians. Prevention of venous thromboembolism:American college of chest physicians evidence-based clinical practice guidelines (8th Edition). CHEST. 2008;133:S381-93.

2. Jacobson BF, Louw S, Mer M, et al. Venous thromboembolismprophylactic and therapeutic practice guideline. South Afr Med J. 2009 Jun;99(6):467-73.

3. Gallagher $M$, Oliver $K$, Hurwitz $M$. Improving the use of venous thromboembolism prophylaxis in an Australian teaching hospital. Qual Saf Health Care. 2009;18:408-12.

4. Clark $\mathrm{P}, \mathrm{Wu} \mathrm{O}$, Greer IA, et al. Venous thrombosis prevention-more than just guidelines. Br J of Haematol. 2008 Feb;149:50-4.

5. Cohen AT, Tapson VF, Bergmann JF, et al. Venous thromboembolism risk and prophylaxis in the acute hospital care setting (ENDORSE study): a multinational cross-sectional study. Lancet. 2008;371(9610):387-94.

6. McKenna GS, Karthikesalingam A, Walsh SR, et al. Prevention of venous thromboembolism: improving practice in surgical patients. Int J of Surg. 2009;7:50-3.

7. Cohn SL. Prophylaxis of venous thromboembolism in the US: improving hospital performance. J of Thromb and Haemost. 2009;7:1437-45.

8. Jacobson BF, Louw S, Bueller $\mathrm{H}$, et al. Venous thromboembolism: prophylactic and therapeutic practice guideline. South Afr Med J. 2013;103(4):261-67.

9. Tooher R, Middleton P, Pham C, et al. A systematic review of strategies to improve prophylaxis for venous thromboembolism in hospitals. Ann of Surg. 2005;241(3):397-415.

10. Brockbank K, Snoxall S, Beaumont A, et al. Implementing a venous thromboembolism (VTE) prevention strategy. Clin Gov an Int J. 2010;15(1):19-28.

11. Veller MG, Pillai J. Lower-limb venous thrombosis. CME.2009;27(7): 306-11.

12. Klein SK, Slim EJ, de Kruif MD, et al. Is chronic HIV infection associated with venous thrombotic disease? A systemic review. Neth J Med. 2005;63:129-36.

13. Ba SA, Badiane SB, Diop SN, et al. A cross-sectional evaluation of venous thrombo-embolism risk and use of venous thrombo-embolism prophylaxis in hospitalised patients in Senegal. Arch of Cardiovasc Dis. 2011;104 (10):493-501.

14. Michota FA. Bridging the gap between evidence and practice in venous thromboembolism prophylaxis: the quality improvement process. J of Gen Int Med. 2007; 22(12):1762-70.

15. Stinnett JM, Pendleton R, Skordos L, et al. Venous thromboembolism prophylaxis in medically ill patients and the development of strategies to improve prophylaxis rates. Am J of Hematol. 2005;78(3):167-72.

Received: 28-01-2014 Accepted: 28-07-2014 